\title{
Robust Guaranteed Cost Observer Design for Singular Markovian Jump Time-Delay Systems with Generally Incomplete Transition Probability
}

\author{
Yanbo Li, ${ }^{1,2}$ Yonggui Kao, ${ }^{2}$ and Jing Xie ${ }^{3}$ \\ ${ }^{1}$ School of Mathematical Sciences, Guangxi Teachers Education University, Guangxi 530001, China \\ ${ }^{2}$ Department of Mathematics, Harbin Institute of Technology, Weihai 264209, China \\ ${ }^{3}$ College of Information Science and Engineering, Ocean University of China, Qingdao 266071, China \\ Correspondence should be addressed to Yonggui Kao; kaoyonggui@sina.com
}

Received 29 November 2013; Revised 6 February 2014; Accepted 10 February 2014; Published 24 March 2014

Academic Editor: Shen Yin

Copyright (c) 2014 Yanbo Li et al. This is an open access article distributed under the Creative Commons Attribution License, which permits unrestricted use, distribution, and reproduction in any medium, provided the original work is properly cited.

\begin{abstract}
This paper is devoted to the investigation of the design of robust guaranteed cost observer for a class of linear singular Markovian jump time-delay systems with generally incomplete transition probability. In this singular model, each transition rate can be completely unknown or only its estimate value is known. Based on stability theory of stochastic differential equations and linear matrix inequality (LMI) technique, we design an observer to ensure that, for all uncertainties, the resulting augmented system is regular, impulse free, and robust stochastically stable with the proposed guaranteed cost performance. Finally, a convex optimization problem with LMI constraints is formulated to design the suboptimal guaranteed cost filters for linear singular Markovian jump time-delay systems with generally incomplete transition probability.
\end{abstract}

\section{Introduction}

Descriptor systems are also referred to as singular systems, implicit systems, generalized state-space systems, or semistate systems and provide convenient and natural representations in the description of economic systems, power systems, robotics, network theory, and circuits systems [1]. The stability for singular system is more complicated than that for nonsingular systems because not only the asymptotic stability but also the system regularity and impulse elimination are needed to be addressed [2-5].

In practice, in many physical systems, such as aircraft control, solar receiver control, power systems, manufacturing systems, networked control systems, air intake systems, and other practical systems, abrupt variations may happen in their structure, due to random failures, repair of components, sudden environmental disturbances, changing subsystem interconnections, or abrupt variations in the operating points of a nonlinear plant [6-19]. Therefore, more and more attention has been paid to the problem of stochastic stability and stochastic admissibility for singular Markovian jump systems (SMJSs) [20-30]. Long et al. [23] derived stochastic admissibility for a class of singular Markovian jump systems with mode-dependent time delays. Wang and Zhang [27] focused on the asynchronous $l_{2}-l_{\infty}$ filtering for discrete-time stochastic Markov jump systems with randomly occurring sensor nonlinearities. However, the TRs in the above mentioned literatures are assumed to be completely known.

In practice, the TRs in some jumping processes are difficult to be precisely estimated due to the cost and some other factors. Therefore, analysis and synthesis problems for normal MJSs with incomplete information on transition probability have attracted more and more attentions [31-49]. Xiong and Lam [32] probed robust $\mathrm{H}_{2}$ control of Markovian jump systems with uncertain switching probabilities. Karan et al. [33] considered the stochastic stability robustness for continuous-time and discrete-time Markovian jump linear systems (MJLSs) with upper bounded TRs. Zhang and Boukas [34] discussed stability and stabilization for the continuous-time MJSs with partly unknown TRs. Lin et al. [38] considered delay-dependent $H_{\infty}$ filtering for discretetime singular Markovian jump systems with time-varying 
delay and partially unknown transition probabilities. Guo and Wang [49] proposed another description for the uncertain TRs, which is called generally uncertain TRs (GUTRs).

On the other hand, state estimation plays an important role in systems and control theory, signal processing, and information fusion $[50,51]$. Certainly, the most widely used estimation method is the well-known Kalman filtering [52, 53]. A common feature in the Kalman filtering is that an accurate model is available. In some applications, however, when the system is subject to parameter uncertainties, the accurate system model is hard to obtain. To overcome this difficulty, the guaranteed cost filtering approach has been proposed to ensure the upper bound of guaranteed cost function [54]. Robust $H_{\infty}$ filtering for uncertain Markovian jump systems with mode-dependent time delays was proposed in [55]. In [56], guaranteed cost and $H_{\infty}$ filtering for timedelay systems were presented in terms of LMIs. However, to the best of our knowledge, there are few considering the robust guaranteed cost observer for a class of linear singular Markovian jump time-delay systems with generally incomplete transition probability, which is still an open problem.

In this paper, based on LMI method, we address the design problem of the robust guaranteed cost observer for a class of uncertain descriptor time-delay systems with Markovian jumping parameters and generally uncertain transition rates. The design problem proposed here is to design a memoryless observer such that for all uncertainties, including generally uncertain transition rates, the resulting augmented system is regular, impulse-free, and robust stochastically stable, and satisfies the proposed guaranteed cost performance.

\section{Problem Formulation}

Consider the following descriptor time-delay systems with Markovian jumping parameters:

$$
\begin{gathered}
E \dot{x}(t)=A\left(r_{t}, t\right) x(t)+A_{d}\left(r_{t}, t\right) x(t-d), \\
y(t)=C\left(r_{t}, t\right) x(t)+C_{d}\left(r_{t}, t\right) x(t-d), \\
x(t)=\varphi(t), \quad \forall t \in[-d, 0],
\end{gathered}
$$

where $x(t) \in R^{n}$ and $y(t) \in R^{r}$ are the state vector and the controlled output, respectively. $d$ represents the state time delay. For convenience, the input terms in system (1) have been omitted. $\varphi(t) \in L_{2}[-d, 0]$ is a continuous vector-valued initial function. The random parameter $\gamma(t)$ represents a continuous-time discrete-state Markov process taking values in a finite set $\mathbb{S}=\{1,2, \ldots, s\}$ and having the transition probability matrix $\Pi=\left[\pi_{i j}\right], i, j \in N$. The transition probability from mode $i$ to mode $j$ is defined by

$$
\operatorname{Pr}\left\{r_{t+\Delta}=j \mid r_{t}=i\right\}= \begin{cases}\pi_{i j} \Delta+o(\Delta), & i \neq j, \\ 1+\pi_{i j} \Delta+o(\Delta), & i=j,\end{cases}
$$

where $\Delta>0$ satisfies $\lim _{\Delta \rightarrow 0}(o(\Delta) / \Delta)=0, \pi_{i j} \geq 0$ is the transition probability from mode $i$ to mode $j$ and satisfies

$$
\pi_{i i}=-\sum_{j=1, j \neq i}^{s} \pi_{i j} \leq 0 .
$$

In this paper, the transition rates of the jumping process are assumed to be partly available; that is, some elements in matrix $\Lambda$ have been exactly known, some have been merely known with lower and upper bounds, and others may have no information to use. For instance, for system (1) with four operation modes, the transition rate matrix might be described by

$$
\Lambda=\left[\begin{array}{ccccc}
\hat{\pi}_{11}+\Delta_{11} & ? & ? & \cdots & ? \\
? & ? & \widehat{\pi}_{23}+\Delta_{23} & \cdots & \widehat{\pi}_{2 s}+\Delta_{2 s} \\
\vdots & \vdots & \vdots & \ddots & \vdots \\
? & \hat{\pi}_{s 2}+\Delta_{s 2} & ? & \cdots & ?
\end{array}\right]
$$

where $\hat{\pi}_{i j}$ and $\Delta_{i j} \in\left[-\sigma_{i j}, \sigma_{i j}\right]\left(\sigma_{i j} \geq 0\right)$ represent the estimate value and estimate error of the uncertain TR $\pi_{i j}$, respectively, where $\widehat{\pi}_{i j}$ and $\sigma_{i j}$ are known. ? represents the complete unknown TR, which means that its estimate value $\widehat{\pi}_{i j}$ and estimate error bound are unknown.

For notational clarity, for all $i \in \mathbb{S}$, the set $U^{i}$ denotes $U^{i}=U_{k}^{i} \cup U_{u k}^{i}$ with $U_{k}^{i}=\{j:$ The estimate value of $\pi_{i j}$ is known for $\left.j \in \mathbb{S}\right\}, U_{u k}^{i}=\{j:$ The estimate value of $\pi_{i j}$ is unknown for $\left.j \in \mathbb{S}\right\}$. Moreover, if $U_{k}^{i} \neq \emptyset$, it is further described as $U_{k}^{i}=\left\{k_{1}^{i}, k_{2}^{i}, \ldots, k_{m}^{i}\right\}$, where $k_{m}^{i} \in \mathbb{N}^{+}$ represents the $m$ th bound-known element with the index $k_{m}^{i}$ in the $i$ th row of matrix $\Pi$. We assume that the known estimate values of the TRs are well defined. That is

Assumption 1. If $U_{k}^{i}=\mathbb{S}$, then $\widehat{\pi}_{i j}-\sigma_{i j} \geq 0$ (for all $j \in$ $\mathbb{S}, j \neq i), \widehat{\pi}_{i i}=-\sum_{j=1, j \neq i}^{N} \widehat{\pi}_{i j}$ and $\sigma_{i i}=-\sum_{j=1, j \neq i}^{s} \sigma_{i j}$.

Assumption 2. If $U_{k}^{i} \neq \mathbb{S}$ and $i \in U_{k}^{i}$, then $\hat{\pi}_{i j}-\sigma_{i j} \geq$ 0 (for all $j \in \mathbb{S}, j \neq i$ ), $\widehat{\pi}_{i i}+\sigma_{i i} \leq 0$ and $\sum_{j \in U_{k}^{i}} \widehat{\pi}_{i j}$.

Assumption 3. If $U_{k}^{i} \neq \mathbb{S}$ and $i \notin U_{k}^{i}$, then $\widehat{\pi}_{i j}-\sigma_{i j} \geq$ 0 (for all $j \in \mathbb{S}$ ).

Remark 4. The above assumption is reasonable, since it is the direct result from the properties of the TRs (e.g., $\pi_{i j} \geq$ 0 (for all $i, j \in \mathbb{S}, j \neq i$ ) and $\pi_{i i}=-\sum_{j=1, j \neq i}^{s} \pi_{i j}$ ). The above description about uncertain TRs is more general than either the MJSs model with bounded uncertain TRs or the MJSs model with partly uncertain TRs. If $U_{u k}^{i}=\emptyset$, for all $i \in \mathbb{S}$, then generally uncertain TR matrix (4) reduces to bounded uncertain TR matrix (5) as follows:

$$
\left[\begin{array}{cccc}
\hat{\pi}_{11}+\Delta_{11} & \hat{\pi}_{12}+\Delta_{12} & \cdots & \widehat{\pi}_{1 s}+\Delta_{1 s} \\
\hat{\pi}_{21}+\Delta_{21} & \widehat{\pi}_{22}+\Delta_{22} & \cdots & \widehat{\pi}_{2 s}+\Delta_{2 s} \\
\vdots & \vdots & \ddots & \vdots \\
\widehat{\pi}_{s 1}+\Delta_{s 1} & \hat{\pi}_{s 2}+\Delta_{s 2} & \cdots & \widehat{\pi}_{s s}+\Delta_{s s}
\end{array}\right]
$$

where $\widehat{\pi}_{i j}-\Delta_{i j} \geq 0$ (for all $j \in \mathbb{S}, j \neq i$ ), $\widehat{\pi}_{i i}=-\sum_{j=1, j \neq i}^{s} \widehat{\pi}_{i j} \leq$ 0 , and $\Delta_{i i}=\sum_{j=1, j \neq i}^{S} \Delta_{i j}$; if $\sigma_{i j}=0$, for all $i \in \mathbb{S}$, for all $j \in$ 
$U_{k}^{i}$, then generally uncertain TR matrix (4) reduces to partly uncertain TR matrix (6) as follows:

$$
\left[\begin{array}{ccccc}
\pi_{11} & ? & ? & \cdots & ? \\
? & ? & \pi_{23} & \cdots & \pi_{2 s} \\
\vdots & \vdots & \vdots & \ddots & \vdots \\
? & \pi_{s 2} & ? & \cdots & ?
\end{array}\right]
$$

Our results in this paper can be applicable to the general Markovian jump systems with bounded uncertain or partly uncertain TR matrix.

$A(\gamma(t), t), A_{d}(\gamma(t), t), C(\gamma(t), t)$, and $C_{d}(\gamma(t), t)$ are matrix functions of the random jumping process $\gamma(t)$. To simplify the notion, the notation $A_{i}(t)$ represents $A(\gamma(t), t)$ when $\gamma(t)=$ $i$. For example, $A_{d}(\gamma(t), t)$ is denoted by $A_{d i}(t)$ and so on. Further, for each $\gamma(t)=i \in N$, it is assumed that the matrices $A_{i}(t), A_{d i}(t), C_{i}(t)$, and $C_{d i}(t)$ can be described by the following form:

$$
\begin{aligned}
A_{i}(t) & =A_{i}+\Delta A_{i}(t), & A_{d i}(t) & =A_{d i}+\Delta A_{d i}(t), \\
C_{i}(t) & =C_{i}+\Delta C_{i}(t), & C_{d i}(t) & =C_{d i}+\Delta C_{d i}(t),
\end{aligned}
$$

where $A_{i}, A_{d i}, C_{i}$ are $C_{d i}$ known real coefficient matrices with appropriate dimensions. Time-varying matrices $\Delta A_{i}(t), \Delta A_{d i}(t), \Delta C_{i}(t)$, and $\Delta C_{d i}(t)$ represent normbounded uncertainties and satisfy

$$
\left[\begin{array}{cc}
\Delta A_{i}(t) & \Delta A_{d i}(t) \\
\Delta C_{i}(t) & \Delta C_{d i}(t)
\end{array}\right]=\left[\begin{array}{l}
M_{1 i} \\
M_{2 i}
\end{array}\right] F_{i}(t)\left[\begin{array}{ll}
N_{1 i} & N_{2 i}
\end{array}\right]
$$

where $M_{1 i}, M_{2 i}, M_{1 i}$, and $N_{2 i}$ are known constant real matrices of appropriate dimensions, which represent the structure of uncertainties, and $F_{i}(t)$ is an unknown matrix function with Lebesgue measurable elements and satisfies $F_{i}(t) F_{i}^{T}(t) \leq$ I.

Further, for convenience, we assume that the system has the same dimension at each mode and the Markov process is irreducible. Consider the following nominal unforced descriptor time-delay system:

$$
\begin{aligned}
E \dot{x}(t) & =A_{i} x(t)+A_{d i} x(t-d), \\
x(t) & =\varphi(t), \quad \forall t \in[-d, 0] .
\end{aligned}
$$

Let $x_{0}, r_{0}$, and $x\left(t, \varphi, r_{0}\right)$ be the initial state, initial mode, and the corresponding solution of the system (9) at time $t$, respectively.

Definition 5. System (9) is said to be stochastically stable if, for all $\varphi(t) \in L_{2}[-d, 0]$ and initial mode $r_{0} \in N$, there exists a matrix $M>0$ such that

$$
\begin{aligned}
& E\left\{\int_{0}^{\infty}\left\|x\left(t, \varphi, r_{0}\right)\right\|^{2} d t \mid r_{0}, x(t)=\varphi(t), t \in[-d, 0]\right\} \\
& \quad \leq x_{0}^{T} M x_{0} .
\end{aligned}
$$

The following definition can be regarded as an extension of the definition in [2].
Definition 6. (1) System (9) is said to be regular if $\operatorname{det}(s E-$ $\left.A_{i}\right), i=1,2, \ldots, s$ are not identically zero.

(2) System (9) is said to be impulse free if $\operatorname{deg}(\operatorname{det}(s E-$ $\left.\left.A_{i}\right)\right)=\operatorname{rank} E_{i}, i=1,2, \ldots, s$.

(3) System (9) is said to be admissible if it is regular, impulse free, and stochastically stable.

The linear memoryless observer under consideration is as follows:

$$
\begin{aligned}
E \dot{\hat{x}}(t) & =K_{1 i} \widehat{x}(t)+K_{2 i} y(t), \\
\widehat{x}_{0} & =0, \quad r(0)=r_{0},
\end{aligned}
$$

where $\hat{x}(t) \in R^{n}$ is the observer state, and the constant matrices $K_{1 i}$ and $K_{2 i}$ are observer parameters to be designed.

Denote the error state $e(t)=x(t)-\widehat{x}(t)$, and the augmented state vector $x_{f}=\left[\begin{array}{ll}x^{T}(t) & e^{T}(t)\end{array}\right]^{T}$. Let $\tilde{x}(t)=L e(t)$ represent the output of the error states, where $L$ is a known constant matrix. Define

$$
\begin{gathered}
A_{f i}=\left[\begin{array}{cc}
A_{i} & 0 \\
A_{i}-K_{1 i}-K_{2 i} C_{i} & K_{1 i}
\end{array}\right], \\
A_{f d i}=\left[\begin{array}{cc}
A_{d i} & 0 \\
A_{d i}-K_{2 i} C_{d i} & 0
\end{array}\right], \quad E_{f}=\left[\begin{array}{cc}
E & 0 \\
0 & E
\end{array}\right], \\
M_{f i}=M_{f 1 i}=\left[\begin{array}{c}
M_{1 i} \\
M_{1 i}-K_{2 i} M_{2 i}
\end{array}\right], \quad N_{f i}=\left[\begin{array}{ll}
N_{1 i} & 0
\end{array}\right], \\
\Delta A_{f i}=M_{f i} F_{i}(t) N_{f i}, \quad N_{f 1 i}=\left[\begin{array}{ll}
N_{2 i} & 0
\end{array}\right], \\
\Delta A_{f d i}=M_{f 1 i} F_{i}(t) N_{f 1 i}, \quad C_{f}=\left[\begin{array}{ll}
0 & L
\end{array}\right]
\end{gathered}
$$

and combine (1) and (11); then we derive the augmented systems as follows:

$$
\begin{array}{r}
E_{f} \dot{x}_{f}(t)=\left(A_{f i}+\Delta A_{f i}\right) x_{f}(t) \\
+\left(A_{f d i}+\Delta A_{f d i}\right) x_{f}(t-d), \\
z(t)=C_{f} x_{f}(t), \\
x_{f 0}(t)=\left[\varphi^{T}(t), \varphi^{T}(t)\right]^{T}, \quad \forall t \in[-d, 0] .
\end{array}
$$

Similar to [5], it is also assumed in this paper that, for all $\varsigma \epsilon$ $[-d, 0]$, there exists a scalar $h>0$ such that $\left\|x_{f}(t+\varsigma)\right\| \leq$ $h\left\|x_{f}(t)\right\|$.

Associated with system (13) is the cost function

$$
\mathscr{J}=\mathbb{E}\left\{\int_{0}^{\infty} z^{T}(t) z(t) d t\right\} .
$$

Definition 7. Consider the augmented system (13), if there exist the observer parameters $K_{1 i}, K_{2 i}$ and a positive scalar $\mathcal{J}^{*}$, for all uncertainties, such that the augmented system (13) is robust, stochastically stable and the value of the cost function (14) satisfies $\mathscr{J} \leq \mathcal{J}^{*}$, then $\mathcal{J}^{*}$ is said to be a robust guaranteed cost and observer (11) is said to be a robust guaranteed cost observer for system (1) with (4).

Problem 8 (robust guaranteed cost observer problem for a class of linear singular Markovian jump time-delay systems 
with generally incomplete transition probability). Given system (1) with GUTR Matrix (4), can we determine an observer (11) with parameters $K_{1 i}$ and $K_{2 i}$ such that the observer is a robust guaranteed cost observer for system (1) with GUTR Matrix (4)?

Lemma 9. Given any real number $\varepsilon$ and any matrix $Q$, the matrix inequality $\varepsilon\left(Q+Q^{T}\right) \leq \varepsilon^{2} T+Q T^{-1} Q^{T}$ holds for any matrix $T>0$.

\section{Main Results}

Theorem 10. Consider the augmented system (13) with GUTR Matrix (4) and the cost function (14). Then the robust guaranteed cost observer (11) with parameters $K_{1 i}$ and $K_{2 i}$ can be designed if there exist matrices $P_{i}, K_{1 i}$, and $K_{2 i}, i=$ $1,2, \ldots, s$, and symmetric positive definite matrix $Q$, satisfying the following LMIs, respectively:

Case 1. If $i \notin U_{k}^{i}$ and $U_{k}^{i}=\left\{k_{1}^{i}, \ldots, k_{m}^{i}\right\}$, there exist a set of symmetric positive definite matrices $T_{i j} \in \mathbb{R}^{n \times n}\left(i \notin U_{k}^{i}, j \in\right.$ $\left.U_{k}^{i}\right)$ such that

$$
\begin{gathered}
E_{f}^{T} P_{i}=P_{i}^{T} E_{f} \geq 0, \\
{\left[\begin{array}{ccc}
\Pi_{i}+C_{f}^{T} C_{f} & P_{i}\left(A_{f d i}+\Delta A_{f d i}\right) & \widehat{N}_{1} \\
\left(A_{f d i}+\Delta A_{f d i}\right)^{T} P_{i} & -Q & 0 \\
* & * & \widehat{N}_{2}
\end{array}\right]<0,} \\
P_{i}-P_{j} \geq 0, \quad \forall j \in U_{u k}^{i}, \quad j \neq i .
\end{gathered}
$$

Case 2. If $i \in U_{k}^{i}, U_{k}^{i}=\left\{k_{1}^{i}, \ldots, k_{m}^{i}\right\}$ and $U_{u k}^{i} \neq \emptyset$, there exist a set of symmetric positive definite matrices $V_{i j l} \in \mathbb{R}^{n \times n}(i, j \in$ $\left.U_{k}^{i}, l \in U_{u k}^{i}\right)$ such that

$$
\begin{gathered}
E_{f}^{T} P_{i}=P_{i}^{T} E_{f} \geq 0 \\
{\left[\begin{array}{ccc}
\Omega_{i}+C_{f}^{T} C_{f} & P_{i}\left(A_{f d i}+\Delta A_{f d i}\right) & \widehat{M}_{1} \\
\left(A_{f d i}+\Delta A_{f d i}\right)^{T} P_{i} & -Q & 0 \\
* & * & \widehat{M}_{2}
\end{array}\right]<0 .}
\end{gathered}
$$

Case 3. If $i \in U_{k}^{i}$ and $U_{u k}^{i}=\emptyset$, there exist a set of symmetric positive definite matrices $W_{i j} \in \mathbb{R}^{n \times n}\left(i, j \in U_{k}^{i}\right)$ such that

$$
\begin{gathered}
E_{f}^{T} P_{i}=P_{i}^{T} E_{f} \geq 0, \\
{\left[\begin{array}{ccc}
\Delta_{i}+C_{f}^{T} C_{f} & P_{i}\left(A_{f d i}+\Delta A_{f d i}\right) & \widehat{L}_{1} \\
\left(A_{f d i}+\Delta A_{f d i}\right)^{T} P_{i} & -Q & 0 \\
* & * & \widehat{L}_{2}
\end{array}\right]<0,}
\end{gathered}
$$

where

$$
\begin{aligned}
\Pi_{i}= & \left(A_{f i}+\Delta A_{f i}\right)^{T} P_{i}+P_{i}\left(A_{f i}+\Delta A_{f i}\right) \\
& +Q+\sum_{j \in U_{k}^{i}} \widehat{\pi}_{i j} E_{f}^{T}\left(P_{j}-P_{i}\right)+\sum_{j \in U_{k}^{i}} \frac{1}{4} \sigma_{i j}^{2} T_{i j},
\end{aligned}
$$

$$
\begin{gathered}
\Omega_{i}=\left(A_{f i}+\Delta A_{f i}\right)^{T} P_{i}+P_{i}\left(A_{f i}+\Delta A_{f i}\right) \\
+Q+\sum_{j \in U_{k}^{i}} \widehat{\pi}_{i j} E_{f}^{T}\left(P_{j}-P_{l}\right)+\sum_{j \in U_{k}^{i}} \frac{1}{4} \sigma_{i j}^{2} V_{i j l}, \\
\Delta_{i}=\left(A_{f i}+\Delta A_{f i}\right)^{T} P_{i}+P_{i}\left(A_{f i}+\Delta A_{f i}\right) \\
+Q+\sum_{j \in \mathbb{S}, j \neq i} \widehat{\pi}_{i j} E_{f}^{T}\left(P_{j}-P_{i}\right)+\sum_{j \in \mathbb{S}, j \neq i} \frac{1}{4} \sigma_{i j}^{2} W_{i j}, \\
\widehat{N}_{1}=\left[E_{f}^{T}\left(P_{i k_{1}^{i}}-P_{i}\right), E_{f}^{T}\left(P_{i k_{2}^{i}}-P_{i}\right), \ldots, E_{f}^{T}\left(P_{i k_{m}^{i}}-P_{i}\right)\right], \\
\widehat{N}_{2}=\operatorname{diag}\left\{-T_{i k_{1}^{i}}, \ldots,-T_{i k_{m}^{i}}\right\}, \\
\widehat{M}_{1}=\left[E_{f}^{T}\left(P_{k_{1}^{i}}-P_{l}\right), E_{f}^{T}\left(P_{k_{2}^{i}}-P_{l}\right), \ldots, E_{f}^{T}\left(P_{k_{m}^{i}}-P_{l}\right)\right], \\
\widehat{M}_{2}=\operatorname{diag}\left\{-V_{i k_{1}^{i} l}, \ldots,-V_{i k_{m}^{i} l}\right\} \\
\widehat{L}_{1}=\left[E_{f}^{T}\left(P_{1}-P_{i}\right), \ldots, E_{f}^{T}\left(P_{i-1}-P_{i}\right),\right. \\
\left.E_{f}^{T}\left(P_{i+1}-P_{i}\right), \ldots, E_{f}^{T}\left(P_{s}-P_{i}\right)\right], \\
\widehat{L}_{2}=\operatorname{diag}\left\{-W_{i 1}, \ldots,-W_{i s}\right\} .
\end{gathered}
$$

Proof. According to Definition 2 and Theorem 1 in [2], we can derive from (15)-(21) that system (13) is regular and impulse free. Let the mode at time $t$ be $i$, and consider the following Lyapunov function with respect to the augmented system (13)

$$
\begin{aligned}
V\left(x_{f}(t), \gamma(t)=i\right)= & x_{f}^{T}(t) E_{f}^{T} P_{i} x_{f}(t) \\
& +\int_{t-d}^{t} x_{f}^{T}(s) Q x_{f}(s) d t
\end{aligned}
$$

where $Q$ is the symmetric positive definite matrix to be chosen, and $P_{i}$ is a matrix satisfying (15)-(21). The weak infinitesimal operator $\mathscr{L}$ of the stochastic process $\left\{\gamma(t), x_{f}(t)\right\}, t \geq 0$, is presented by

$$
\begin{aligned}
& \mathscr{L} V\left(x_{f}(t), \gamma(t)=i\right) \\
& \begin{aligned}
\lim _{\Delta \rightarrow 0} \frac{1}{\Delta}[ & E_{f}\{V(x(t+\Delta), \gamma(t+\Delta)) x(t), \gamma(t)=i\} \\
& -V(x(t), \gamma(t)=i)] \\
=x_{f}^{T}(t)[ & {\left[A_{f i}+\Delta A_{f i}\right)^{T} P_{i}+P_{i}\left(A_{f i}+\Delta A_{f i}\right) } \\
& \left.+\sum_{j=1}^{s} \pi_{i j} E_{f}^{T} P_{j}+Q\right] x_{f}(t) \\
+ & 2 x_{f}^{T}(t) P_{i}\left(A_{f 1 i}+\Delta A_{f 1 i}\right) x_{f}(t-d) \\
- & x_{f}^{T}(t-d) Q x_{f}(t-d) .
\end{aligned}
\end{aligned}
$$


Case $1\left(i \notin U_{k}^{i}\right)$. Note that in this case $\sum_{j \in U_{u k}^{i}, j \neq i} \pi_{i j}=$ $-\sum_{j \in \mathscr{U}_{k}^{i}, j \neq i} \pi_{i j}-\pi_{i i}$ and $\pi_{i j} \geq 0, j \in U_{u k}^{i}, j \neq i$; then from (24), we have

$$
\begin{aligned}
& x_{f}^{T}(t)\left[\sum_{j=1}^{s} \pi_{i j} E_{f}^{T} P_{j}\right] x_{f}(t) \\
& =x_{f}^{T}(t)\left[\sum_{j \in U_{k}^{i}} \pi_{i j} E_{f}^{T} P_{j}+\sum_{j \in U_{u k}^{i}, j \neq i} \pi_{i j} E_{f}^{T} P_{j}+\pi_{i i} E_{f}^{T} P_{j}\right] x_{f}(t) \\
& =x_{f}^{T}(t)\left[\sum_{j \in U_{k}^{i}} \pi_{i j} E_{f}^{T} P_{j}+\left(-\pi_{i i}-\sum_{j \in U_{k}^{i}} \pi_{i j}\right) E_{f}^{T} P_{i}\right. \\
& \left.+\pi_{i i} E_{f}^{T} P_{i}\right] x_{f}(t) \\
& =x_{f}^{T}(t) E_{f}^{T}\left[\sum_{j \in U_{k}^{i}} \pi_{i j}\left(P_{j}-P_{i}\right)\right] x_{f}(t) \\
& =x_{f}^{T}(t) E_{f}^{T}\left[\sum_{j \in U_{k}^{i}} \hat{\pi}_{i j}\left(P_{j}-P_{i}\right)+\sum_{j \in U_{k}^{i}} \Delta_{i j}\left(P_{j}-P_{i}\right)\right] x_{f}(t) .
\end{aligned}
$$

On the other hand, in view of Lemma 9, we have

$$
\begin{aligned}
& \sum_{j \in U_{k}^{i}} \Delta_{i j} E_{f}^{T}\left(P_{j}-P_{i}\right) \\
& \quad=\sum_{j \in U_{k}^{i}}\left[\frac{1}{2} \Delta_{i j} E_{f}^{T}\left(P_{j}-P_{i}\right)+\frac{1}{2} \Delta_{i j} E_{f}^{T}\left(P_{j}-P_{i}\right)\right] \\
& \leq \sum_{j \in U_{k}^{i}}\left[\left(\frac{1}{2} \Delta_{i j}\right)^{2} T_{i j}+E_{f}^{T}\left(P_{j}-P_{i}\right) T_{i j}^{-1}\left(P_{j}-P_{i}\right) E_{f}\right] \\
& \leq \sum_{j \in U_{k}^{i}}\left[\frac{1}{4} \sigma_{i j}^{2} T_{i j}+E_{f}^{T}\left(P_{j}-P_{i}\right) T_{i j}^{-1}\left(P_{j}-P_{i}\right) E_{f}\right] .
\end{aligned}
$$

Case $2\left(i \in U_{k}^{i}\right.$ and $\left.U_{u k}^{i} \neq \emptyset\right)$. Because of $U_{k}^{i}=\left\{k_{1}^{i}, \ldots, k_{m}^{i}\right\}$ and $U_{u k}^{i}=\left\{u_{1}^{i}, \ldots, u_{s-m}^{i}\right\}$, there must be $l \in U_{u k}^{i}$ so that $E_{f}^{T} P_{l} \geq$ $E_{f}^{T} P_{j}$ (for all $j \in U_{u k}^{i}$ ):

$$
\begin{aligned}
& x_{f}^{T}(t)\left[\sum_{j=1}^{s} \pi_{i j} E_{f}^{T} P_{j}\right] x_{f}(t) \\
& \quad \leq x_{f}^{T}(t)\left[\sum_{j \in U_{k}^{i}} \pi_{i j} E_{f}^{T} P_{j}-\left(\sum_{j \in U_{k}^{i}, j \neq i} \pi_{i j}\right) E_{f}^{T} P_{l}\right] x_{f}(t) \\
& \quad=x_{f}^{T}(t) E_{f}^{T}\left[\sum_{j \in U_{k}^{i}} \pi_{i j}\left(P_{j}-P_{l}\right)\right] x_{f}(t)
\end{aligned}
$$

$$
=x_{f}^{T}(t) E_{f}^{T}\left[\sum_{j \in U_{k}^{i}} \hat{\pi}_{i j}\left(P_{j}-P_{l}\right)+\sum_{j \in U_{k}^{i}} \Delta_{i j}\left(P_{j}-P_{l}\right)\right] x_{f}(t) .
$$

By using Lemma 9, we have

$$
\begin{aligned}
& \sum_{j \in U_{k}^{i}} \Delta_{i j} E_{f}^{T}\left(P_{j}-P_{l}\right) \\
& \quad=\sum_{j \in U_{k}^{i j}}\left[\frac{1}{2} \Delta_{i j} E_{f}^{T}\left(P_{j}-P_{l}\right)+\frac{1}{2} \Delta_{i j} E_{f}^{T}\left(P_{j}-P_{l}\right)\right] \\
& \quad \leq \sum_{j \in U_{k}^{i}}\left[\left(\frac{1}{2} \Delta_{i j}\right)^{2} V_{i j l}+E_{f}^{T}\left(P_{j}-P_{l}\right) V_{i j l}^{-1}\left(P_{j}-P_{l}\right) E_{f}^{T}\right] \\
& \quad \leq \sum_{j \in U_{k}^{i}}\left[\frac{1}{4} \sigma_{i j}^{2} V_{i j l}+E_{f}^{T}\left(P_{j}-P_{l}\right) V_{i j l}^{-1}\left(P_{j}-P_{l}\right) E_{f}^{T}\right] .
\end{aligned}
$$

Case $3\left(i \in U_{k}^{i}\right.$ and $\left.U_{u k}^{i}=\emptyset\right)$. Consider

$$
\begin{aligned}
& x_{f}^{T}(t)\left[\sum_{j=1}^{s} \pi_{i j} E_{f}^{T} P_{j}\right] x_{f}(t) \\
& =x_{f}^{T}(t) E_{f}^{T}\left[\sum_{j=1, j \neq i}^{s} \pi_{i j}\left(P_{j}-P_{i}\right)\right] x_{f}(t) \\
& =x_{f}^{T}(t) E_{f}^{T}\left[\sum_{j=1, j \neq i}^{s} \hat{\pi}_{i j}\left(P_{j}-P_{i}\right)\right. \\
& \left.\quad+\sum_{j=1, j \neq i}^{s} \Delta_{i j}\left(P_{j}-P_{i}\right)\right] x_{f}(t) .
\end{aligned}
$$

Case 1. Substituting (25) and (26) into (24), it results in

$$
\mathscr{L} V \leq \Lambda^{T}(t) \Phi(i) \Lambda(t),
$$

where $\Lambda^{T}(t)=\left[x_{f}^{T}(t), x_{f}^{T}(t-d)\right]$ and

$$
\Phi_{i}=\left[\begin{array}{ccc}
\Pi_{i}+C_{f}^{T} C_{f} & P_{i}\left(A_{f d i}+\Delta A_{f d i}\right) & \widehat{N}_{1} \\
\left(A_{f d i}+\Delta A_{f d i}\right)^{T} P_{i} & -Q & 0 \\
* & * & \widehat{N}_{2}
\end{array}\right] .
$$

Case 2. Substituting (27) and (28) into (24), it results in

$$
\mathscr{L} V \leq \Lambda^{T}(t) \Psi(i) \Lambda(t),
$$

where $\Lambda^{T}(t)=\left[x_{f}^{T}(t), x_{f}^{T}(t-d)\right]$ and

$$
\Psi_{i}=\left[\begin{array}{ccc}
\Omega_{i}+C_{f}^{T} C_{f} & P_{i}\left(A_{f d i}+\Delta A_{f d i}\right) & \widehat{M}_{1} \\
\left(A_{f d i}+\Delta A_{f d i}\right)^{T} P_{i} & -Q & 0 \\
* & * & \widehat{M}_{2}
\end{array}\right] .
$$


Case 3. Substituting (29) into (24), we get

$$
\mathscr{L} V \leq \Lambda^{T}(t) \Gamma(i) \Lambda(t),
$$

where $\Lambda^{T}(t)=\left[x_{f}^{T}(t), x_{f}^{T}(t-d)\right]$ and

$$
\Gamma_{i}=\left[\begin{array}{ccc}
\Delta_{i}+C_{f}^{T} C_{f} & P_{i}\left(A_{f d i}+\Delta A_{f d i}\right) & \widehat{L}_{1} \\
\left(A_{f d i}+\Delta A_{f d i}\right)^{T} P_{i} & -Q & 0 \\
* & * & \widehat{L}_{2}
\end{array}\right]
$$

Similar to [5], using Dynkin's formula, we drive for each $i \in N$ :

$$
\lim _{T \rightarrow \infty} \mathbb{E}\left\{\int_{0}^{T} x_{f}^{T}(t) x_{f}(t) d t \mid \varphi_{f}, \gamma_{0}=i\right\} \leq x_{f 0}^{T} M x_{f 0} .
$$

By Definition 5, it is easy to see that the augmented system (13) is stochastically stable. Furthermore, from (16), (19), and (21), we have

$$
\mathscr{L} V \leq-x_{f}^{T}(t) C_{f}^{T} C_{f} x_{f}(t)<0 .
$$

On the other hand, we have

$$
\begin{aligned}
\mathscr{J} & =\mathbb{E}\left\{\int_{0}^{\infty} x_{f}^{T}(t) C_{f}^{T} C_{f} x_{f}(t) d t\right\}<-\int_{0}^{\infty} \mathscr{L} V d t \\
& =-\mathbb{E}\left\{\lim _{t \rightarrow \infty} V(x(t), \gamma(t))\right\}+V\left(x_{0}, \gamma_{0}\right) .
\end{aligned}
$$

As the augmented system (13) is stochastically stable, it follows from (38) that $J<V\left(x_{f 0}, r_{0}\right)$. From Definition 7, it is concluded that a robust guaranteed cost for the augmented system (13) can be given by $J^{*}=x_{f 0}^{T}(t) E_{f r_{0}}^{T} P\left(r_{0}\right) x_{f 0}+$ $\int_{-d}^{0} x_{f}^{T}(t) Q x_{f}(t) d t$.

In the following, based on the above sufficient condition, the design of robust guaranteed cost observers can be turned into the solvability of a system of LMIs.

Theorem 11. Consider system (13) with GUTR Matrix (4) and the cost function (14). If there exist matrices $Y_{1 i}$ and $Y_{2 i}, i=$ $1,2, \ldots, s$ positive scalars $\varepsilon_{i}, i=1,2, \ldots, s$, symmetric positive definite matrix $Q$, and the full rank matrices $P_{2 i}$, and matrices $P_{i}=\operatorname{diag}\left(P_{1 i}, P_{2 i}\right), i=1,2, \ldots, s$, satisfying the following LMIs, respectively.

Case 1. If $i \notin U_{k}^{i}$ and $U_{k}^{i}=\left\{k_{1}^{i}, \ldots, k_{m}^{i}\right\}$, a set of positive definite matrices $T_{i j} \in \mathbb{R}^{n \times n}\left(i \notin U_{k}^{i}, j \in U_{k}^{i}\right)$ exist such that

$$
\begin{gathered}
E_{f}^{T} P_{i}=P_{i}^{T} E_{f} \geq 0, \\
{\left[\begin{array}{cccc}
\phi_{1 i} & \phi_{2 i} & \bar{N}_{1} & \phi_{3 i} \\
\phi_{2 i}^{T} & -Q & 0 & 0 \\
\bar{N}_{1}^{T} & 0 & \bar{N}_{2} & 0 \\
\phi_{3 i}^{T} & 0 & 0 & -\varepsilon_{i} I
\end{array}\right]<0,} \\
P_{i}-P_{j} \geq 0, \quad \forall j \in U_{u k}^{i}, j \neq i .
\end{gathered}
$$

Case 2. If $i \in U_{k}^{i}\left(U_{k}^{i}=\left\{k_{1}^{i}, \ldots, k_{m}^{i}\right\}\right)$ and $U_{u k}^{i} \neq \emptyset$, a set of positive definite matrices $V_{i j l} \in \mathbb{R}^{n \times n}\left(i, j \in U_{k}^{i}, l \in U_{u k}^{i}\right)$ exist such that

$$
E_{f}^{T} P_{i}=P_{i}^{T} E_{f} \geq 0
$$

$$
\left[\begin{array}{cccc}
\varphi_{1 i} & \varphi_{2 i} & \bar{M}_{1} & \varphi_{3 i} \\
\varphi_{2 i}^{T} & -Q & 0 & 0 \\
\bar{M}_{1}^{T} & 0 & \bar{M}_{2} & 0 \\
\varphi_{3 i}^{T} & 0 & 0 & -\varepsilon_{i} I
\end{array}\right]<0 .
$$

Case 3. If $i \in U_{k}^{i}$ and $U_{u k}^{i}=\emptyset$, a set of positive definite matrices $W_{i j} \in \mathbb{R}^{n \times n}\left(i, j \in U_{k}^{i}\right)$ exist such that

$$
E_{f i}^{T} P_{i}=P_{i}^{T} E_{f i} \geq 0
$$

$$
\left[\begin{array}{cccc}
\psi_{1 i} & \psi_{2 i} & \bar{L}_{1} & \psi_{3 i} \\
\psi_{2 i}^{T} & -Q & 0 & 0 \\
\bar{L}_{1}^{T} & 0 & \bar{L}_{2} & 0 \\
\psi_{3 i}^{T} & 0 & 0 & -\varepsilon_{i} I
\end{array}\right]<0,
$$

where

$$
\begin{aligned}
& \phi_{1 i}=\varphi_{1 i}=\psi_{1 i} \\
& =\left[\begin{array}{cc}
P_{1 i} A_{i}+A_{i}^{T} P_{1 i} & A_{i}^{T} P_{2 i}-Y_{1 i}^{T}-C_{i}^{T} Y_{2 i}^{T} \\
P_{2 i} A_{i}-Y_{1 i}-Y_{2 i} C_{i} & Y_{1 i}^{T}+Y_{1 i}
\end{array}\right] \\
& +Q+C_{f}^{T} C_{f}+\sum_{j \in U_{k}^{i}} \widehat{\pi}_{i j} E_{f}^{T}\left(P_{j}-P_{i}\right)+\sum_{j \in U_{k}^{i}} \frac{1}{4} \sigma_{i j}^{2} T_{i j}, \\
& \phi_{2 i}=\varphi_{2 i}=\psi_{2 i}=\left[\begin{array}{cc}
P_{1 i} A_{i} & 0 \\
P_{2 i} A_{i}-Y_{1 i}-Y_{2 i} C_{i} & 0
\end{array}\right] \text {, } \\
& \phi_{3 i}=\varphi_{3 i}=\psi_{3 i}=\left[\begin{array}{c}
P_{1 i} M_{1 i} \\
P_{2 i} M_{1 i}-Y_{1 i} M_{1 i}-Y_{2 i} M_{2 i}
\end{array}\right] \text {, } \\
& \bar{N}_{1}=\left[E_{f}^{T}\left(P_{k_{1}^{i}}-P_{i}\right), E_{f}^{T}\left(P_{k_{2}^{i}}-P_{i}\right), \ldots, E_{f}^{T}\left(P_{k_{m}^{i}}-P_{i}\right)\right] \text {, } \\
& \bar{N}_{2}=\operatorname{diag}\left\{-T_{i k_{1}^{i}}, \ldots,-T_{i k_{m}^{i}}\right\}, \\
& \bar{M}_{1}=\left[E_{f}^{T}\left(P_{k_{1}^{i}}-P_{i}\right), E_{f}^{T}\left(P_{k_{2}^{i}}-P_{i}\right), \ldots, E_{f}^{T}\left(P_{k_{m}^{i}}-P_{i}\right)\right] \text {, } \\
& \bar{M}_{2}=\operatorname{diag}\left\{-V_{i k_{1}^{i}}, \ldots,-V_{i k_{m}^{i} l}\right\} \text {, } \\
& \bar{L}_{1}=\left[E_{f}^{T}\left(P_{1}-P_{i}\right), \ldots, E_{f}^{T}\left(P_{i-1}-P_{i}\right)\right. \text {, } \\
& \left.E_{f}^{T}\left(P_{i+1}-P_{i}\right), \ldots, E_{f}^{T}\left(P_{s}-P_{i}\right)\right], \\
& \bar{L}_{2}=\operatorname{diag}\left\{-W_{i 1}, \ldots,-W_{i s}\right\} \text {. }
\end{aligned}
$$


Then a suitable robust guaranteed cost observer in the form of (11) has parameters as follows:

$$
K_{1 i}=P_{1 i}^{-1} Y_{1 i}, \quad K_{2 i}=P_{2 i}^{-1} Y_{2 i}
$$

and $J^{*}$ is a robust guaranteed cost for system (13) with GUTR Matrix (4).

Proof. Define

$$
\begin{gathered}
A_{i}^{1}=\left[\begin{array}{ccc}
A_{f i}^{T} P_{i}+P_{i} A_{f i}+Q+\sum_{j \in U_{k}^{i}} \hat{\pi}_{i j}\left(P_{j}-P_{i}\right)+\sum_{j \in U_{k}^{i}} \frac{1}{4} \sigma_{i j}^{2} T_{i j}+C_{f}^{T} C_{f} & P_{i} A_{f d i} \bar{N}_{1} \\
A_{f d i}^{T} P_{i} & -Q & 0 \\
* & * & \bar{N}_{2}
\end{array}\right], \\
A_{i}^{2}=\left[\begin{array}{cccc}
A_{f i}^{T} P_{i}+P_{i} A_{f i}+Q+\sum_{j \in U_{k}^{i}} \hat{\pi}_{i j}\left(P_{j}-P_{i}\right)+\sum_{j \in U_{k}^{i}} \frac{1}{4} \sigma_{i j}^{2} V_{i j l}+C_{f}^{T} C_{f} & P_{i} A_{f d i} & \bar{M}_{1} \\
A_{f d i}^{T} P_{i} & -Q & 0 \\
* & * & \bar{M}_{2}
\end{array}\right], \\
A_{i}^{3}=\left[\begin{array}{cccc}
A_{f i}^{T} P_{i}+P_{i} A_{f i}+Q+\sum_{j \in U_{k}^{i}} \widehat{\pi}_{i j}\left(P_{j}-P_{i}\right)+\sum_{j \in U_{k}^{i}} \frac{1}{4} \sigma_{i j}^{2} W_{i j}+C_{f}^{T} C_{f} & P_{i} A_{f d i} & \bar{L}_{1} \\
A_{f d i}^{T} P_{i} & -Q & 0 \\
* & * & \bar{L}_{2}
\end{array}\right]<0 .
\end{gathered}
$$

Then (16) is equivalent to

$$
\begin{aligned}
A_{i}^{1}+ & {\left[\begin{array}{c}
P_{i} M_{f i} \\
0 \\
0
\end{array}\right] F_{i}\left[\begin{array}{lll}
N_{f i} & N_{f 1 i} & 0
\end{array}\right] } \\
& +\left[\begin{array}{lll}
N_{f i} & F_{f 1 i} & 0
\end{array}\right]^{T} F_{i}^{T}\left[\begin{array}{c}
P_{i} M_{f i} \\
0 \\
0
\end{array}\right]^{T}<0 .
\end{aligned}
$$

Then (19) is equivalent to

$$
\begin{aligned}
A_{i}^{2}+ & {\left[\begin{array}{c}
P_{i} M_{f i} \\
0 \\
0
\end{array}\right] F_{i}\left[\begin{array}{lll}
N_{f i} & N_{f 1 i} & 0
\end{array}\right] } \\
& +\left[\begin{array}{lll}
N_{f i} & F_{f 1 i} & 0
\end{array}\right]^{T} F_{i}^{T}\left[\begin{array}{c}
P_{i} M_{f i} \\
0 \\
0
\end{array}\right]^{T}<0 .
\end{aligned}
$$

Then (21) is equivalent to

$$
\begin{aligned}
A_{i}^{3}+ & {\left[\begin{array}{c}
P_{i} M_{f i} \\
0 \\
0
\end{array}\right] F_{i}\left[\begin{array}{lll}
N_{f i} & N_{f 1 i} & 0
\end{array}\right] } \\
& +\left[\begin{array}{lll}
N_{f i} & F_{f 1 i} & 0
\end{array}\right]^{T} F_{i}^{T}\left[\begin{array}{c}
P_{i} M_{f i} \\
0 \\
0
\end{array}\right]^{T}<0 .
\end{aligned}
$$

By applying Lemma 2.4 in [57], (50), (51), and (52) hold for all uncertainties $F_{i}$ satisfying $F_{i}^{T} F_{i}<I$ if and only if there exist positive scalars $\varepsilon_{i}, i=1,2, \ldots, s$, such that

$$
\begin{aligned}
& A_{i}^{1}+\varepsilon_{i}^{-1}\left[\begin{array}{c}
P_{i} M_{f i} \\
0 \\
0
\end{array}\right]\left[\begin{array}{c}
P_{i} M_{f i} \\
0 \\
0
\end{array}\right]^{T} \\
& +\varepsilon_{i}\left[\begin{array}{lll}
N_{f i} & F_{f 1 i} & 0
\end{array}\right]^{T}\left[\begin{array}{lll}
N_{f i} & F_{f 1 i} & 0
\end{array}\right]<0, \\
& A_{i}^{2}+\varepsilon_{i}^{-1}\left[\begin{array}{c}
P_{i} M_{f i} \\
0 \\
0
\end{array}\right]\left[\begin{array}{c}
P_{i} M_{f i} \\
0 \\
0
\end{array}\right]^{T} \\
& +\varepsilon_{i}\left[\begin{array}{lll}
N_{f i} & F_{f 1 i} & 0
\end{array}\right]^{T}\left[\begin{array}{lll}
N_{f i} & F_{f 1 i} & 0
\end{array}\right]<0, \\
& A_{i}^{3}+\varepsilon_{i}^{-1}\left[\begin{array}{c}
P_{i} M_{f i} \\
0 \\
0
\end{array}\right]\left[\begin{array}{c}
P_{i} M_{f i} \\
0 \\
0
\end{array}\right]^{T} \\
& +\varepsilon_{i}\left[\begin{array}{lll}
N_{f i} & F_{f 1 i} & 0
\end{array}\right]^{T}\left[\begin{array}{lll}
N_{f i} & F_{f 1 i} & 0
\end{array}\right]<0 .
\end{aligned}
$$

Let $P_{i}=\operatorname{diag}\left(P_{1 i}, P_{2 i}\right)$, and using (47), we can conclude from Schur complement results that the above matrix inequalities are equivalent to the coupled LMIs (40), (43), and (45). It further follows from Theorem 10 that $J^{*}$ is a robust guaranteed cost for system (13) with (4).

Remark 12. The solution of LMIs (39)-(45) parameterizes the set of the proposed robust guaranteed cost observers. This parameterized representation can be used to design the guaranteed cost observer with some additional performance constraints. By applying the methods in [14], the suboptimal 
guaranteed cost observer can be determined by solving a certain optimization problem. This is the following theorem.

Theorem 13. Consider system (13) with GUTR Matrix (4) and the cost function (14), and suppose that the initial conditions $r_{0}$ and $x_{f 0}$ are known; if the following optimization problem

$$
\begin{aligned}
& \min _{\mathrm{Q}, P_{1 i}, P_{2 i}, \mathcal{\varepsilon}_{i}, Y_{1 i} \text { and } Y_{2 i}} J^{*} \\
& \text { s.t. LMIs (39)- }(45)
\end{aligned}
$$

has a solution $Q, P_{1 i}, P_{2 i}, \varepsilon_{i}, Y_{1 i}$, and $Y_{2 i}, i=1,2, \ldots, s$, then the observer (11) is a suboptimal guaranteed cost observer for system (1), where $J^{*}=x_{f 0}^{T} E_{f r_{0}}^{T} P\left(r_{0}\right) x_{f 0}+$ $\operatorname{tr}\left(\int_{-d}^{0} x_{f 0}(t) x_{f 0}(t) x_{f 0}^{T} d t Q\right)$.

Proof. It follows from Theorem 11 that the observer (11) constructed in terms of the solution $Q, P_{1 i}, P_{2 i}, \varepsilon_{i}, Y_{1 i}$, and $Y_{2 i}, i=1,2, \ldots, s$, is a robust guaranteed cost observer. By noting that

$$
\begin{aligned}
\int_{-d}^{0} x_{f 0}^{T}(t) Q x_{f 0}(t) d t & =\int_{-d}^{0} \operatorname{tr}\left(x_{f 0}^{T}(t) Q x_{f 0}(t)\right) d t \\
& =\operatorname{tr}\left(\int_{-d}^{0} x_{f 0}^{T}(t) x_{f 0}(t) d t Q\right),
\end{aligned}
$$

it follows that the suboptimal guaranteed cost observer problem is turned into the minimization problem (55).

Remark 14. Theorem 13 gives the suboptimal guaranteed cost observer conditions of a class of linear Markovian jumping time-delay systems with generally incomplete transition probability and LMI constraints, which can be easily solved by the LMI toolbox in MATLAB.

\section{Numerical Example}

In this section, a numerical example is presented to demonstrate the effectiveness of the method mentioned in Theorem 11. Consider a 2-dimensional system (1) with 3 Markovian switching modes. In this numerical example, the singular system matrix is set as $E=\left[\begin{array}{ll}1 & 0 \\ 0 & 0\end{array}\right]$, and the 3-mode transition rate matrix is $\Lambda=\left[\begin{array}{ccc}-3.2 & ? & ? \\ ? & ? & 2 \\ 1.5 & 2.1 & -3.6\end{array}\right]$, where $\Delta_{11}, \Delta_{31} \in$ $[-0.15,0.15] ; \Delta_{23}, \Delta_{33} \in[-0.12,0.12]$ and $\Delta_{32} \in[-0.1,0.1]$. The other system matrices are as follows.

For mode $i=1$, there are

$$
\begin{array}{ccc}
A_{1}=\left[\begin{array}{cc}
-3.2 & 0.65 \\
1 & 0.2
\end{array}\right], & A_{d 1}=\left[\begin{array}{cc}
0.2 & 0.5 \\
1 & -0.68
\end{array}\right], \\
C_{1}=\left[\begin{array}{cc}
1.2 & 0.65 \\
-6.5 & 1.9 \\
-0.21 & -1.8
\end{array}\right], & C_{d 1}=\left[\begin{array}{cc}
-3.6 & -1.05 \\
2.1 & 0.96 \\
0.21 & -0.86
\end{array}\right], \\
M_{11}=\left[\begin{array}{c}
-0.2 \\
0.8
\end{array}\right], & M_{21}=\left[\begin{array}{c}
0.25 \\
0.875 \\
-2
\end{array}\right], \\
N_{11}=\left[\begin{array}{ll}
-1.2 & 3.1
\end{array}\right], & N_{21}=[-0.69-4.2] .
\end{array}
$$

For mode $i=2$, there are

$$
\begin{aligned}
& A_{2}=\left[\begin{array}{cc}
-1 & 6 \\
2 & -3.6
\end{array}\right], \quad A_{d 2}=\left[\begin{array}{cc}
-3.1 & -1.6 \\
3 & 0.75
\end{array}\right] \\
& C_{2}=\left[\begin{array}{cc}
9 & -2.5 \\
0.35 & -2 \\
3.6 & -1.8
\end{array}\right], \quad C_{d 2}=\left[\begin{array}{cc}
0.89 & -6 \\
-1.2 & 0.9 \\
-2.4 & 6
\end{array}\right] \text {, } \\
& M_{12}=\left[\begin{array}{c}
2.3 \\
-4
\end{array}\right], \quad M_{22}=\left[\begin{array}{c}
0.75 \\
-3.6 \\
2.5
\end{array}\right] \text {, } \\
& N_{12}=\left[\begin{array}{ll}
-7.2 & -6
\end{array}\right], \quad N_{22}=\left[\begin{array}{ll}
1 & 2
\end{array}\right] \text {. }
\end{aligned}
$$

For mode $i=3$, there are

$$
\begin{array}{cc}
A_{3}=\left[\begin{array}{cc}
-10.6 & 2.9 \\
-0.3 & 3.6
\end{array}\right], & A_{d 3}=\left[\begin{array}{cc}
-5.6 & -1.2 \\
-3 & 4.5
\end{array}\right], \\
C_{3}=\left[\begin{array}{cc}
-3 & -0.36 \\
0.15 & -1.8 \\
0.9 & -5
\end{array}\right], & C_{d 3}=\left[\begin{array}{cc}
-1.65 & 5 \\
-1.2 & 2.65 \\
-0.98 & -5.6
\end{array}\right], \\
M_{13}=\left[\begin{array}{l}
-8.2 \\
-0.3
\end{array}\right], & M_{23}=\left[\begin{array}{c}
-0.52 \\
2.5 \\
-3.6
\end{array}\right], \\
N_{13}=[1.05-5], & N_{23}=[-7.2-1.26] .
\end{array}
$$

Then, we set the error state matrix $L=\left[\begin{array}{cc}-45 & 0.6 \\ 2 & -6\end{array}\right]$, and the positive scalars in Theorem 11 are $\varepsilon_{1}=0.2, \varepsilon_{2}=0.15, \varepsilon_{3}=$ 0.32 . According to the definitions of augmented state matrices in (12), we can easily obtain the following parameter matrices in Theorem 11 by MATLAB

$$
\begin{aligned}
& Y_{11}=\left[\begin{array}{cc}
-8452.1006 & 0.0127 \\
0.0127 & 8450.9001
\end{array}\right] \text {, } \\
& Y_{21}=\left[\begin{array}{ccc}
0.02 & 0.1291 & 0.1435 \\
-2.3520 & -0.4080 & -0.8106
\end{array}\right] \text {, } \\
& Y_{12}=\left[\begin{array}{cc}
-17.0991 & 26.9626 \\
26.9626 & -24.6750
\end{array}\right] \text {, } \\
& Y_{22}=\left[\begin{array}{ccc}
-20.0744 & -13.2941 & 52.9388 \\
21.6893 & 18.0120 & -50.6693
\end{array}\right] \text {, } \\
& Y_{13}=\left[\begin{array}{cc}
-675.1329 & 22.4456 \\
22.4456 & -897.6976
\end{array}\right] \text {, } \\
& Y_{23}=\left[\begin{array}{ccc}
-13.6021 & -146.1726 & 54.0500 \\
-3.4324 & -19.5125 & -10.3068
\end{array}\right] \text {, } \\
& P_{1}=\left[\begin{array}{cccc}
6.3029 & 0 & 0 & 0 \\
0 & 4.8620 & 0 & 0 \\
0 & 0 & 2.2914 & 0 \\
0 & 0 & 0 & 0.3169
\end{array}\right] \text {, }
\end{aligned}
$$




$$
\begin{aligned}
& P_{2}=\left[\begin{array}{cccc}
0.8914 & 0 & 0 & 0 \\
0 & 1.2505 & 0 & 0 \\
0 & 0 & 7.3629 & 0 \\
0 & 0 & 0 & 3.0056
\end{array}\right] \\
& P_{3}=\left[\begin{array}{cccc}
3.0265 & 0 & 0 & 0 \\
0 & 0.2156 & 0 & 0 \\
0 & 0 & 0.8965 & 0 \\
0 & 0 & 0 & 1.0002
\end{array}\right] \text {, } \\
& Q=\left[\begin{array}{cccc}
0.5000 & 0 & 0 & 0 \\
0 & 0.5001 & 0 & 0 \\
0 & 0 & 0.5001 & 0 \\
0 & 0 & 0 & 0.5001
\end{array}\right] \text {, } \\
& T_{11}=\left[\begin{array}{cccc}
3417.3214 & -870.7765 & 0 & 0 \\
-870.7765 & 416.7216 & 0 & 0 \\
0 & 0 & 2226.3598 & -320.7456 \\
0 & 0 & -320.7456 & 1226.3101
\end{array}\right] \text {, } \\
& T_{23} \\
& =\left[\begin{array}{cccc}
3775.3231 & -2799.9330 & 0 & 0 \\
-2799.9330 & 2810.7685 & 0 & 0 \\
0 & 0 & 10690.7366 & -10743.2750 \\
0 & 0 & -10743.2750 & 10855.5053
\end{array}\right] \text {, } \\
& T_{31}=\left[\begin{array}{cccc}
951.8504 & -539.9245 & 0 & 0 \\
-539.9245 & 896.2029 & 0 & 0 \\
0 & 0 & 1477.3012 & -207.7540 \\
0 & 0 & -207.7540 & 1479.1256
\end{array}\right] \text {, } \\
& T_{32}=\left[\begin{array}{cccc}
2161.7695 & -1209.4164 & 0 & 0 \\
-1209.4164 & 2037.1205 & 0 & 0 \\
0 & 0 & 1.4786 & -0.9283 \\
0 & 0 & -0.9283 & 1.4794
\end{array}\right] \text {, } \\
& T_{33}=\left[\begin{array}{cccc}
1493.9313 & -839.8780 & 0 & 0 \\
-839.8780 & 1407.3689 & 0 & 0 \\
0 & 0 & 147.8123 & -133.6452 \\
0 & 0 & -133.6452 & 245.9347
\end{array}\right] \text {, } \\
& V_{11}=\left[\begin{array}{cccc}
1.6650 & 0 & 0 & 0 \\
0 & 1.6650 & 0 & 0 \\
0 & 0 & 1.6650 & 0 \\
0 & 0 & 0 & 1.6650
\end{array}\right] \\
& W_{31}=\left[\begin{array}{cccc}
1.5426 & 0 & 0 & 0 \\
0 & 1.6650 & 0 & 0 \\
0 & 0 & 1.6662 & 0 \\
0 & 0 & 0 & 1.6650
\end{array}\right] \text {, } \\
& W_{32}=\left[\begin{array}{cccc}
1.5428 & 0 & 0 & 0 \\
0 & 1.6650 & 0 & 0 \\
0 & 0 & 1.6622 & 0 \\
0 & 0 & 0 & 1.6650
\end{array}\right] \text {. }
\end{aligned}
$$

Therefore, we can design a linear memoryless observer as (11) with the constant matrices

$$
\begin{gathered}
K_{11}=P_{11}^{-1} Y_{11}=\left[\begin{array}{cc}
-1340.9860 & 0.0020 \\
0.0026 & 1738.1530
\end{array}\right], \\
K_{21}=P_{21}^{-1} Y_{21}=\left[\begin{array}{ccc}
0.0087 & 0.0563 & 0.0626 \\
-7.4219 & -1.2875 & -2.5579
\end{array}\right], \\
K_{12}=P_{12}^{-1} Y_{12}=\left[\begin{array}{cc}
-19.1823 & 30.2475 \\
21.5615 & -19.7321
\end{array}\right], \\
K_{22}=P_{22}^{-1} Y_{22}=\left[\begin{array}{ccc}
-2.7264 & -1.8056 & 7.1899 \\
7.2163 & 5.9928 & -16.8583
\end{array}\right], \\
K_{13}=P_{13}^{-1} Y_{13}=\left[\begin{array}{ccc}
-223.1402 & 7.4186 \\
104.1076 & -4163.7180
\end{array}\right], \\
K_{23}=P_{23}^{-1} Y_{23}=\left[\begin{array}{ccc}
-15.1724 & -163.0481 & 60.2900 \\
-3.4317 & -19.5086 & -10.3047
\end{array}\right] .
\end{gathered}
$$

Finally, the observer (11) with the above parameter matrices for this numerical example is a suboptimal guaranteed cost observer by Theorems 11 and 13 .

\section{Conclusions}

In this paper, the robust guaranteed cost observer problem for a class of uncertain descriptor time-delay systems with Markovian jumping parameters and generally uncertain transition rates is studied by using LMI method. In this GUTR singular model, each transition rate can be completely unknown or only its estimate value is known. The parameter's uncertainty is time varying and is assumed to be norm-bounded. Memoryless guaranteed cost observers are designed in terms of a set of linear coupled matrix inequalities. The suboptimal guaranteed cost observer is designed by solving a certain optimization problem. Our results can be applicable to the general Markovian jump systems with bounded uncertain or partly uncertain TR matrix.

\section{Conflict of Interests}

The authors declare that there is no conflict of interests regarding the publication of this paper.

\section{Acknowledgments}

This research is supported by the National Natural Science Foundations of China (10701020, 10971022, 60973048, 61272077, 60974025, 60673101, and 60939003), NCET-08-0755, National 863 Plan Project (2008 AA04Z401, 2009AA043404), SDPW IMZQWH010016, the Natural Science Foundation of Shandong Province (no. ZR2012FM006), the Scientific and Technological Project of Shandong Province (no. 2007GG3WZ04016), and the Natural Science Foundation of Guangxi Autonomous Region (no. 2012GXNSFBA053003). 


\section{References}

[1] L. Dai, Singular Control Systems, Springer, Berlin, Germany, 1989.

[2] S. Xu, P. V. Dooren, R. Ştefan, and J. Lam, "Robust stability and stabilization for singular systems with state delay and parameter uncertainty," IEEE Transactions on Automatic Control, vol. 47, no. 7, pp. 1122-1128, 2002.

[3] M. Fu and G. Duan, "Robust guaranteed cost observer for uncertain descriptor time-delay systems with Markovian jumping parameters," Acta Automatica Sinica, vol. 31, pp. 479-483, 2005.

[4] Y. Q. Xia, E.-K. Boukas, P. Shi, and J. H. Zhang, "Stability and stabilization of continuous-time singular hybrid systems," Automatica, vol. 45, no. 6, pp. 1504-1509, 2009.

[5] S. Xu and J. Lam, Robust Control and Filtering of Singular Systems, Springer, Berlin, Germany, 2006.

[6] Y.-Y. Cao and J. Lam, "Robust $H_{\infty}$ control of uncertain Markovian jump systems with time-delay," IEEE Transactions on Automatic Control, vol. 45, no. 1, pp. 77-83, 2000.

[7] Y. Li and Y. Kao, "Stability of stochastic reaction-diffusion systems with Markovian switching and impulsive perturbations," Mathematical Problems in Engineering, vol. 2012, Article ID 429568, 13 pages, 2012.

[8] X. Mao and C. Yuan, Stochastic Differential Equations with Markovian Switching, Imperial College Press, London, UK, 2006.

[9] E.-K. Boukas, Stochastic Switching Systems: Analysis and Design, Birkhäuser, Basel, Switzerland, 2005.

[10] Y. Kao, C. Wang, and L. Zhang, "Delay-dependent exponential stability of impulsive markovian jumping Cohen-Grossberg neural networks with reaction-diffusion and mixed delays," Neural Processing Letters, vol. 38, no. 3, pp. 321-346, 2013.

[11] Y.-G. Kao, J.-F. Guo, C.-H. Wang, and X.-Q. Sun, "Delaydependent robust exponential stability of Markovian jumping reaction-diffusion Cohen-Grossberg neural networks with mixed delays," Journal of the Franklin Institute, vol. 349, no. 6, pp. 1972-1988, 2012.

[12] Y. Kao, C. Wang, F. Zha, and H. Cao, "Stability in mean of partial variables for stochastic reaction-diffusion systems with Markovian switching," Journal of the Franklin Institute, vol. 351, no. 1, pp. 500-512, 2014.

[13] N. Kumaresan and P. Balasubramaniam, "Optimal control for stochastic nonlinear singular system using neural networks," Computers \& Mathematics with Applications, vol. 56, no. 9, pp. 2145-2154, 2008.

[14] L. Yu and J. Chu, "An LMI approach to guaranteed cost control of linear uncertain time-delay systems," Automatica, vol. 35, no. 6, pp. 1155-1159, 1999.

[15] S. Yin, H. Luo, and S. Ding, "Real-time implementation of faulttolerant control systems with performance optimization," IEEE Transactions on Industrial Electronics, vol. 64, no. 5, pp. 24022411, 2014.

[16] S. Yin, G. Wang, and H. Karimi, "Data-driven design of robust fault detection system for wind turbines," Mechatronics, 2013.

[17] S. Yin, S. X. Ding, A. H. A. Sari, and H. Hao, "Data-driven monitoring for stochastic systems and its application on batch process," International Journal of Systems Science, vol. 44, no. 7, pp. 1366-1376, 2013.

[18] S. Yin, S. Ding, A. Haghani, H. Hao, and P. Zhang, "A comparison study of basic datadriven fault diagnosis and process monitoring methods on the benchmark Tennessee Eastman process," Journal of Process Control, vol. 22, no. 9, pp. 1567-1581, 2012.

[19] Y. G. Kao and C. H. Wang, "Global stability analysis for stochastic coupled reaction-diffusion systems on networks," Nonlinear Analysis. Real World Applications, vol. 14, no. 3, pp. 1457-1465, 2013.

[20] E. K. Boukas, "Stabilization of stochastic singular nonlinear hybrid systems," Nonlinear Analysis. Theory, Methods \& Applications, vol. 64, no. 2, pp. 217-228, 2006.

[21] E.-K. Boukas, Control of Singular Systems with Random Abrupt Changes, Springer, Berlin, Germany, 2008.

[22] Y. Ding, H. Zhu, S. Zhong, and Y. Zeng, "Exponential meansquare stability of time-delay singular systems with Markovian switching and nonlinear perturbations," Applied Mathematics and Computation, vol. 219, no. 4, pp. 2350-2359, 2012.

[23] S. Long, S. Zhong, and Z. Liu, "Stochastic admissibility for a class of singular Markovian jump systems with modedependent time delays," Applied Mathematics and Computation, vol. 219, no. 8, pp. 4106-4117, 2012.

[24] Z. Wu, P. Shi, H. Su, and J. Chu, "Asynchronous $I_{2}-I_{\infty}$ filtering for discrete-time stochastic Markov jump systems with randomly occurred sensor nonlinearities," Automatica, vol. 50, no. 1, pp. 180-186, 2014.

[25] Z. Wu, P. Shi, H. Su, and J. Chu, "Stochastic synchronization of Markovian jump neural networks with time-varying delay using sampled-data," IEEE Transactions on Cybernetics, vol. 43, no. 6, pp. 1796-1806, 2013.

[26] S. Ma and E.-K. Boukas, "Guaranteed cost control of uncertain discrete-time singular Markov jump systems with indefinite quadratic cost," International Journal of Robust and Nonlinear Control, vol. 21, no. 9, pp. 1031-1045, 2011.

[27] G. Wang and Q. Zhang, "Robust control of uncertain singular stochastic systems with Markovian switching via proportionalderivative state feedback," IET Control Theory \& Applications, vol. 6, no. 8, pp. 1089-1096, 2012.

[28] J. Wang, H. Wang, A. Xue, and R. Lu, "Delay-dependent $H_{\infty}$ control for singular Markovian jump systems with time delay," Nonlinear Analysis. Hybrid Systems, vol. 8, pp. 1-12, 2013.

[29] L. Wu, X. Su, and P. Shi, "Sliding mode control with bounded $L_{2}$ gain performance of Markovian jump singular time-delay systems," Automatica, vol. 48, no. 8, pp. 1929-1933, 2012.

[30] Z.-G. Wu, J. H. Park, H. Su, and J. Chu, "Stochastic stability analysis for discrete-time singular Markov jump systems with timevarying delay and piecewise-constant transition probabilities," Journal of the Franklin Institute, vol. 349, no. 9, pp. 2889-2902, 2012.

[31] J. Xiong, J. Lam, H. Gao, and D. W. C. Ho, "On robust stabilization of Markovian jump systems with uncertain switching probabilities," Automatica, vol. 41, no. 5, pp. 897-903, 2005.

[32] J. Xiong and J. Lam, "Robust $H_{2}$ control of Markovian jump systems with uncertain switching probabilities," International Journal of Systems Science, vol. 40, no. 3, pp. 255-265, 2009.

[33] M. Karan, P. Shi, and C. Y. Kaya, "Transition probability bounds for the stochastic stability robustness of continuousand discrete-time Markovian jump linear systems," Automatica, vol. 42, no. 12, pp. 2159-2168, 2006.

[34] L. Zhang and E.-K. Boukas, "Stability and stabilization of Markovian jump linear systems with partly unknown transition probabilities," Automatica, vol. 45, no. 2, pp. 463-468, 2009. 
[35] B. Du, J. Lam, Y. Zou, and Z. Shu, "Stability and stabilization for Markovian jump time-delay systems with partially unknown transition rates," IEEE Transactions on Circuits and Systems. I. Regular Papers, vol. 60, no. 2, pp. 341-351, 2013.

[36] L. Zhang and J. Lam, "Necessary and sufficient conditions for analysis and synthesis of Markov jump linear systems with incomplete transition descriptions," IEEE Transactions on Automatic Control, vol. 55, no. 7, pp. 1695-1701, 2010.

[37] Y. Guo and F. Zhu, "New results on stability and stabilization of Markovian jump systems with partly known transition probabilities," Mathematical Problems in Engineering, vol. 2012, Article ID 869842, 11 pages, 2012.

[38] J. Lin, S. Fei, and J. Shen, "Delay-dependent $H_{\infty}$ filtering for discrete-time singular Markovian jump systems with timevarying delay and partially unknown transition probabilities," Signal Processing, vol. 91, no. 2, pp. 277-289, 2011.

[39] J. Tian, Y. Li, J. Zhao, and S. Zhong, "Delay-dependent stochastic stability criteria for Markovian jumping neural networks with mode-dependent time-varying delays and partially known transition rates," Applied Mathematics and Computation, vol. 218, no. 9, pp. 5769-5781, 2012.

[40] Y. Wei, J. Qiu, H. R. Karimi, and M. Wang, "A new design $H_{\infty}$ filtering for contrinuous-time Markovian jump systems with time-varying delay and partially accessible mode information," Signal Processing, vol. 93, pp. 2392-2407, 2013.

[41] L. Zhang, E.-K. Boukas, and J. Lam, "Analysis and synthesis of Markov jump linear systems with time-varying delays and partially known transition probabilities," IEEE Transactions on Automatic Control, vol. 53, no. 10, pp. 2458-2464, 2008.

[42] L. Zhang and E.-K. Boukas, "Mode-dependent $H_{\infty}$ filtering for discrete-time Markovian jump linear systems with partly unknown transition probabilities," Automatica, vol. 45, no. 6 , pp. 1462-1467, 2009.

[43] L. Zhang and E.-K. Boukas, "Mode-dependent $H_{\infty}$ control for discrete-time Markovian jump linear systems with partly unknown transition probabilities," International Journal of Robust and Nonlinear Control, vol. 19, no. 8, pp. 868-883, 2009.

[44] Y. Zhang, Y. He, M. Wu, and J. Zhang, "Stabilization for Markovian jump systems with partial information on transition probability based on free-connection weighting matrices," Automatica, vol. 47, no. 1, pp. 79-84, 2011.

[45] G. Zong, D. Yang, L. Hou, and Q. Wang, "Robust finite-time $H_{\infty}$ control for Markovian jump systems with partially known transition probabilities," Journal of the Franklin Institute, vol. 350, no. 6, pp. 1562-1578, 2013.

[46] Y. Ding, H. Zhu, S. Zhong, and Y. Zhang, " $L_{2}-L_{\infty}$ filtering for Markovian jump systems with time-varying delays and partly unknown transition probabilities," Communications in Nonlinear Science and Numerical Simulation, vol. 17, no. 7, pp. 3070-3081, 2012.

[47] Q. Ma, S. Xu, and Y. Zou, "Stability and synchronization for Markovian jump neural networks with partly unknown transition probabilities," Neurocomputing, vol. 74, pp. 34033411, 2011.

[48] E. Tian, D. Yue, and G. Wei, "Robust control for Markovian jump systems with partially known transition probabilities and nonlinearities," Journal of the Franklin Institute, vol. 350, no. 8 , pp. 2069-2083, 2013.

[49] Y. Guo and Z. Wang, "Stability of Markovian jump systems with generally uncertain transition rates," Journal of the Franklin Institute, vol. 350, no. 9, pp. 2826-2836, 2013.
[50] L. Zhang, " $H_{\infty}$ estimation for discrete-time piecewise homogeneous Markov jump linear systems," Automatica, vol. 45, no. 11, pp. 2570-2576, 2009.

[51] Z. Wu, H. Su, and J. Chu, "state estimation for discrete Markovian jumping neural networks with time delay," Neurocomputing, vol. 73, pp. 2247-2254, 2010.

[52] I. R. Petersen and A. V. Savkin, Robust Kalman Filtering for Signals and Systems with Large Uncertainties, Birkhäuser, Boston, Mass, USA, 1999.

[53] B. D. O. Anderson and J. B. Moore, Optimal Filtering, PrenticeHall, Upper Saddle River, NJ, USA, 1979.

[54] I. R. Petersen and D. C. McFarlane, "Optimal guaranteed cost control and filtering for uncertain linear systems," IEEE Transactions on Automatic Control, vol. 39, no. 9, pp. 1971-1977, 1994.

[55] S. Xu, T. Chen, and J. Lam, "Robust $H^{\infty}$ filtering for uncertain Markovian jump systems with mode-dependent time delays," IEEE Transactions on Automatic Control, vol. 48, no. 5, pp. 900907, 2003.

[56] J. H. Kim, D. Oh, and H. Park, "Guaranteed cost and $H_{\infty}$ filtering for time-delay systems," in Proceeding of the American Control Conference, pp. 4014-4018, IEEE, Arlington, Va, USA, 2001.

[57] L. Xie, "Output feedback $H_{\infty}$ control of systems with parameter uncertainty," International Journal of Control, vol. 63, no. 4, pp. 741-750, 1996. 


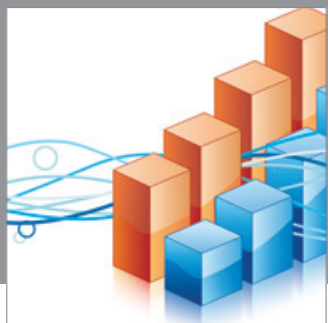

Advances in

Operations Research

mansans

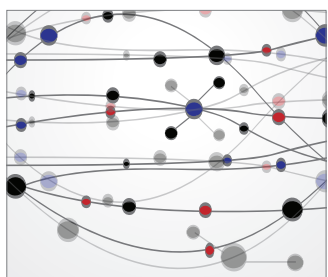

The Scientific World Journal
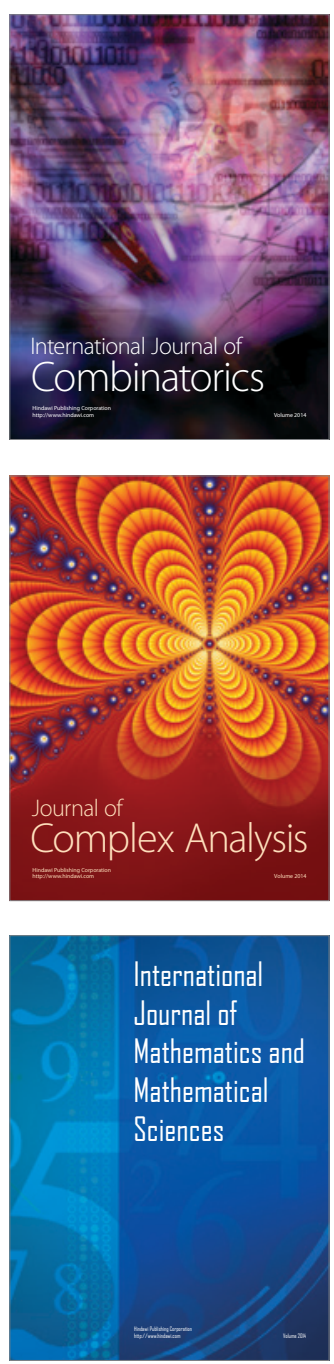
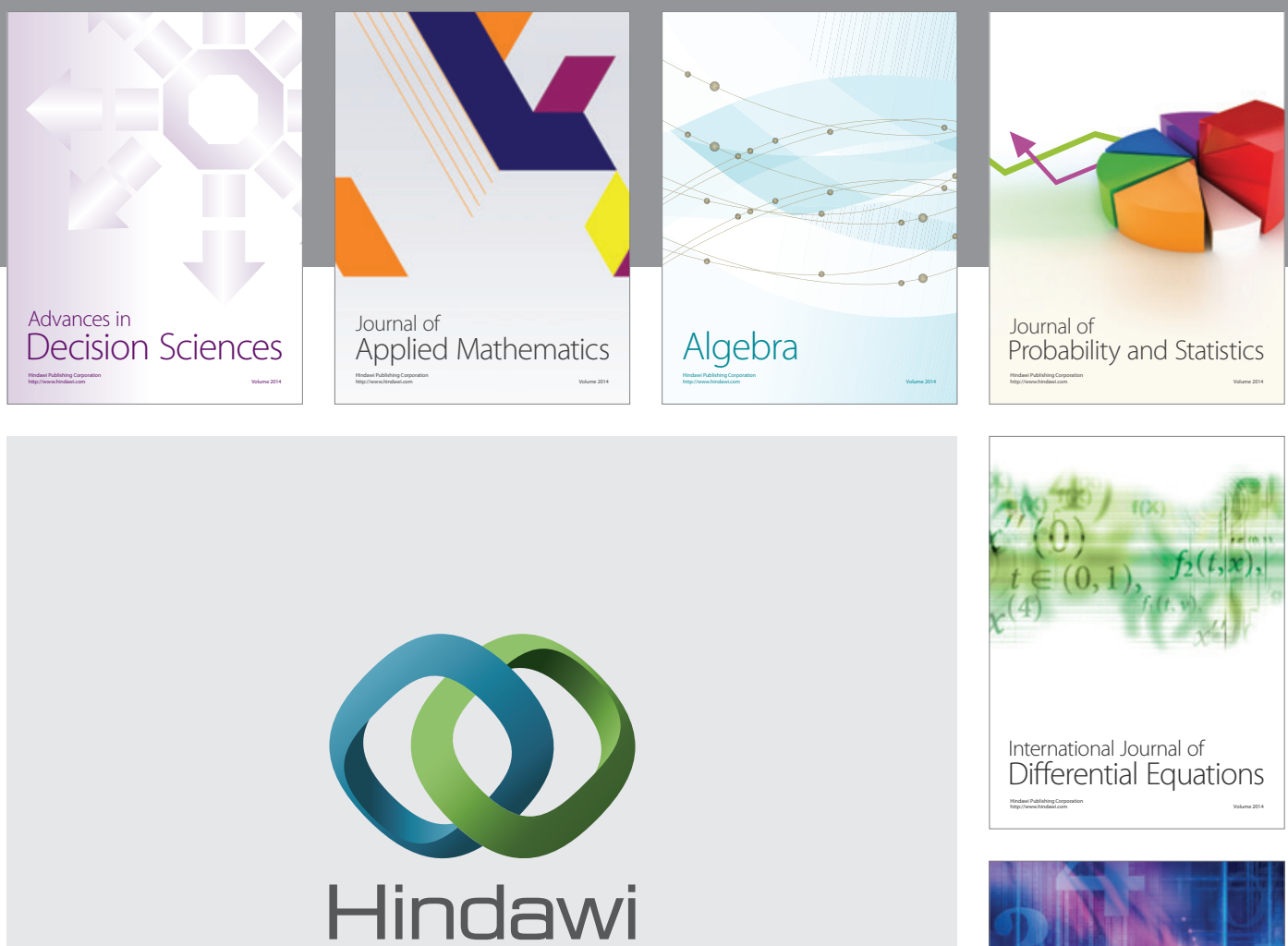

Submit your manuscripts at http://www.hindawi.com
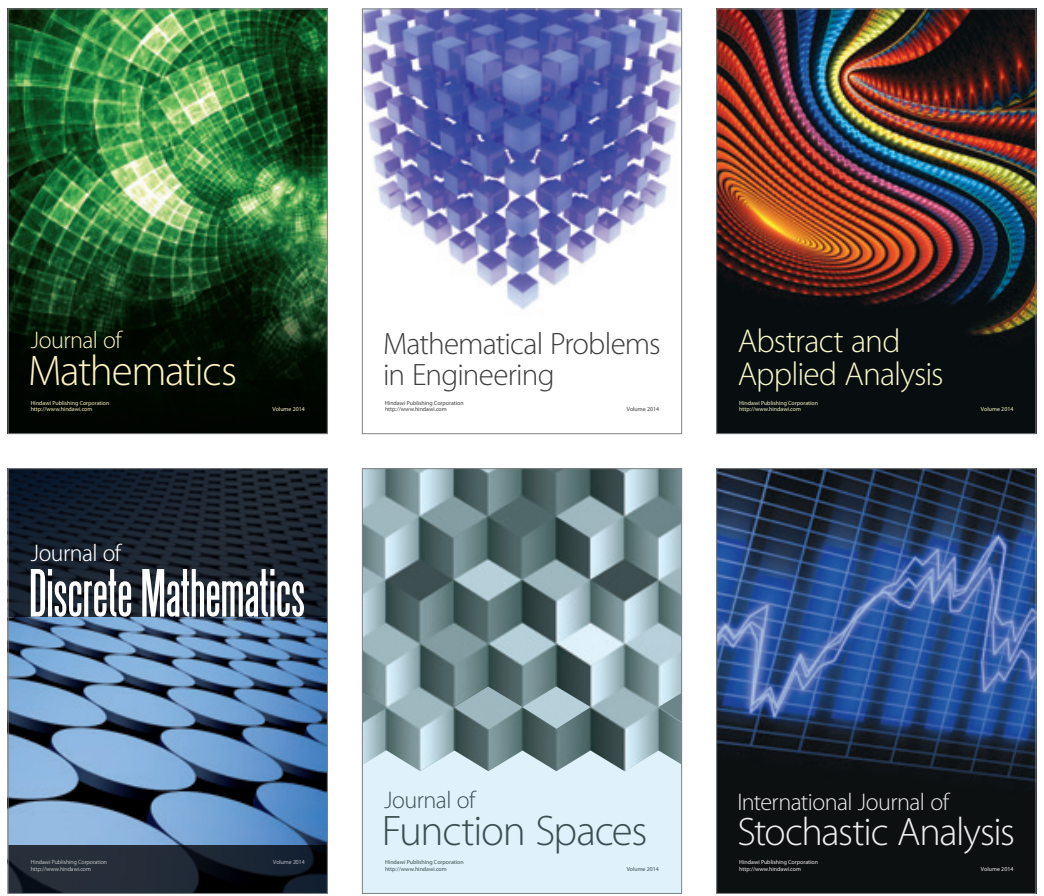

Journal of

Function Spaces

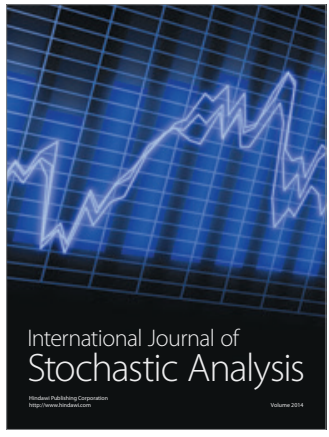

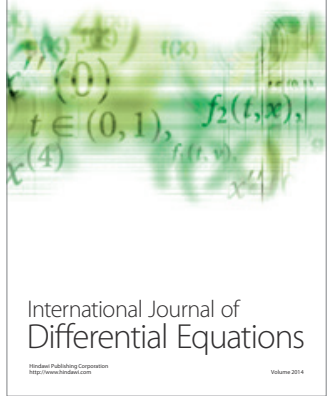
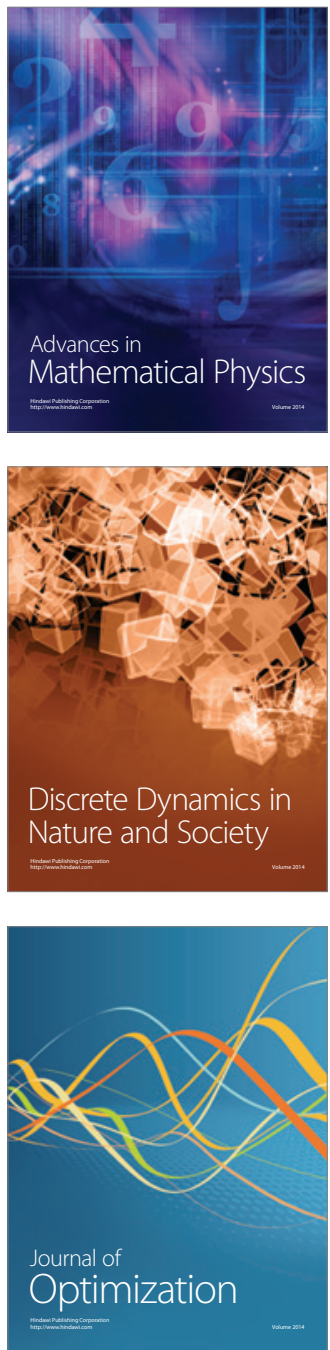